EPJ Web of Conferences 41, 01002 (2013)

DOI: $10.1051 /$ epjconf/20134101002

(C) Owned by the authors, published by EDP Sciences, 2013

\title{
Tunable High Harmonic Generation driven by a Visible Optical Parametric Amplifier
}

\author{
G. Cirmi ${ }^{1,2,{ }^{*}}$, C.-J. Lai ${ }^{1}$, S.-W. Huang ${ }^{1}$, E. Granados ${ }^{1,3}$, A. Sell ${ }^{1}$, J. Moses ${ }^{1}$, K.-H. Hong ${ }^{1}$, \\ P. Keathley ${ }^{\prime}$, and F. X. Kärtner ${ }^{1,2}$
}

${ }^{1}$ Department of Electrical Engineering and Computer Science and Research Laboratory of Electronics, Massachusetts Institute of Technology, 02139 Cambridge MA, USA

${ }^{2}$ Center for Free-Electron Laser Science, DESY and University of Hamburg, 22607 Hamburg, Germany

${ }^{3}$ IKERBASQUE, Basque Foundation for Science, 48011, Bilbao, Spain

*giovanni.cirmi@cfel.de

\begin{abstract}
We studied high-harmonic generation (HHG) in Ar, Ne and He gas jets using a broadly tunable, high-energy optical parametric amplifier (OPA) in the visible wavelength range. We optimized the noncollinear OPA to deliver tunable, femtosecond pulses with 200-500 $\mu \mathrm{J}$ energy at $1-\mathrm{kHz}$ repetition rate with excellent spatiotemporal properties, suitable for HHG experiments. By tuning the central wavelength of the OPA while keeping energy, duration and beam size constant, we experimentally studied the scaling law of conversion efficiency and cut-off energy with the driver wavelength in argon and helium respectively. Our measurements show a $\lambda^{-5.9 \pm 0.9}$ wavelength dependence of the conversion efficiency and a $\lambda^{1.7 \pm 0.2}$ dependence of the HHG cut-off photon energy over the full visible range in agreement with previous experiments of near- and mid-IR wavelengths. By tuning the central wavelength of the driver source and changing the gas, the high order harmonic spectra in the extreme ultraviolet cover the full range of photon energy between $\sim 25 \mathrm{eV}$ and $\sim 100 \mathrm{eV}$. Due to the high coherence intrinsic in HHG, as well as the broad and continuous tunability in the extreme UV range, a high energy, high repetition rate version of this source might be an ideal seed for free electron lasers.
\end{abstract}

\section{Introduction}

Free electron lasers (FELs) are revolutionary extreme UV (EUV) sources due to their very high photon flux allowing for pump-probe and imaging experiments. FELs typically operate by selfamplified spontaneous emission, which inevitably contributes to the poor coherence, pulse-to-pulse timing jitter and poor electric field reproducibility of the output pulses, limiting the time resolution in pump-probe experiments to around $100 \mathrm{fs}$. In order to improve their coherence and stability, there has been much recent interest in the direct seeding of high photon flux EUV sources with high harmonic generation (HHG) in noble gases, due to the excellent temporal and spatial coherence of HHG pulses. Pulse energies in the nJ range [1] are required to effectively seed FELs in the tens of $\mathrm{eV}$ photon energy range to overcome spontaneous emission and transfer the beneficial HHG coherence properties to the FEL output light.

This is an Open Access article distributed under the terms of the Creative Commons Attribution License 2.0, which permits unrestricted use, distribution, and reproduction in any medium, provided the original work is properly cited. 
In recent years, the wavelength scaling of cut-off energy and conversion efficiency of HHG has been extensively studied with long-wavelength pulses in the infrared region [2], mainly because it is beneficial to extend the cut-off energy of the produced harmonics. However, the HHG efficiency dramatically decreases for longer driver wavelengths. Visible drivers are better suited for the generation of high-flux EUV radiation below $\sim 100 \mathrm{eV}$, because they guarantee higher conversion efficiencies than infrared drivers.

In this work we developed a high-energy visible optical parametric amplifier (OPA), allowing for a broad tunability from 470 to $640 \mathrm{~nm}$. We then used the visible pulses from the OPA to drive HHG, obtained by focusing the driver pulses into $\mathrm{Ar}, \mathrm{Ne}$ and $\mathrm{He}$ atoms, and experimentally studied the scaling laws for cut-off energy and conversion efficiency, extending the results from the IR to the visible wavelengths. We also show full tunability of the generated EUV pulses, between $25 \mathrm{eV}$ and $100 \mathrm{eV}$.

\section{Tunable OPA in the visible range}

The visible OPA has been for many years a workhorse of ultrafast spectroscopy at femtosecond time scales, where $\mu \mathrm{J}$-level energies are needed. To achieve high pulse energy, short pulse duration, high spatiotemporal quality, and wavelength tunability in a visible light source, we designed an OPA based on white light continuum (WLC) generation and an amplified Ti:sapphire laser. The design is similar to that of Ref. [3], but contains key differences based on our priorities of high spatiotemporal quality, crucial for HHG driving, and compatibility with a Ti:sapphire laser of 35-fs pulse duration.

The experimental setup is composed of a commercial Ti:sapphire system, a 3-stage visible OPA, and a HHG setup. The pulse duration of the Ti:sapphire pulses is 35-fs, allowing compatibility with the most commonly used laser systems for strong-field physics. Because of the short pulse duration, the number of refractive optical elements needs to be minimized to avoid the material dispersion and self-phase modulation in the high energy beam paths. For the same reason we used a relatively large number (3) of amplification stages. In this way we could lower the gain per each stage (in particular the second and the third), ensuring optimum spatiotemporal beam properties.

We generate WLC in a sapphire plate to seed the first OPA stage. In this stage we focused the pump at $400 \mathrm{~nm}$, obtained through second harmonic generation from Ti:sapphire, and the WLC seed in a type-I BBO in a noncollinear configuration generating a visible signal output with pulse energies up to $5 \mu \mathrm{J}$. We compressed the output pulses by a pair of prisms at Brewster's angle. Before the amplification in the second and third stages, we tilted the 2-mJ, 400-nm pump beam front to match pump and signal wave fronts. The energy after the final stage was 200-550 $\mu \mathrm{J}$. We measured the pulse duration with a background free autocorrelator based on self-diffraction, and the durations were around $30 \mathrm{fs}$. The OPA presented a good beam quality $\left(\mathrm{M}^{2}=1.9\right)$.

\section{Tunable HHG}
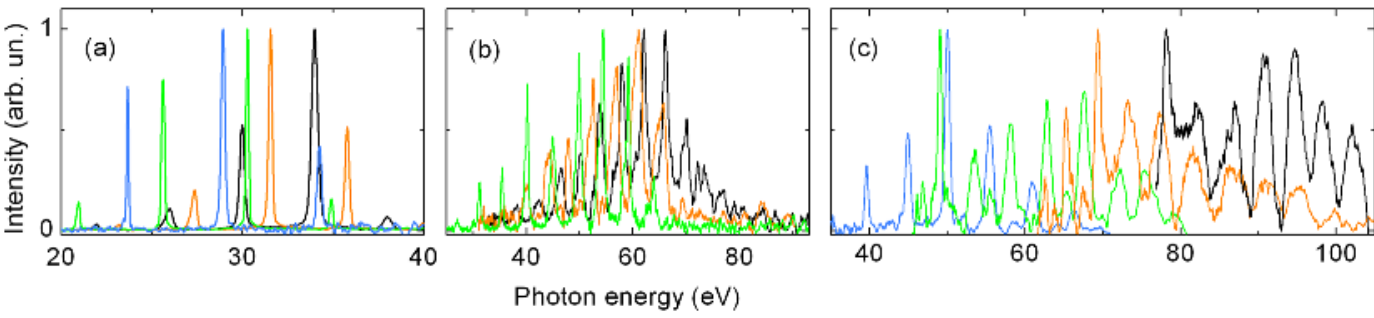

Fig. 1. Linear scale HHG spectra for $\mathrm{Ar}(\mathrm{a}), \mathrm{Ne}(\mathrm{b}), \mathrm{He}(\mathrm{c})$.

In the HHG experiments we focused the OPA beam into jets of different gases ( $\mathrm{Ar}, \mathrm{Ne}$ and $\mathrm{He}$ ), and measured the total HHG output signal and its spectrum. Tunability is a crucial characteristic for a 
seed source, because it makes it possible to finely match the photon energy of the seed with the photon energy of the FEL. Figure 1 shows several spectra measured while tuning the driver wavelength in $\mathrm{Ar}$, Ne and He gases.

The HHG spectra show a broad continuous tunability, allowing for the production of any photon energy between 25 and $100 \mathrm{eV}$. We exploited the HHG tunability by adjusting the OPA central wavelength and changing the gas. A source similar to this, after proper scaling of the energy and of the repetition rate, can be used for seeding EUV FELs, which have typically the same spectral range as the HHG source demonstrated here, and this source is eventually a good candidate for seeding hard-X-ray FELs using cascade schemes.

\section{Efficiency and cutoff energy scaling}

For the experimental study on the cut-off energy and conversion efficiency, we changed the central wavelength of the OPA while keeping the energy, the intensity and the spot size nearly constant, and measured the cut-off energy and conversion efficiency vs. driver wavelength. We focused the study on argon gas for the efficiency study because of the higher efficiency, and on helium gas for the cutoff study because its high ionization potential allows the highest cut-off energy among all gases. We drove HHG with the OPA tuned at different central wavelengths, and also with the light at $800 \mathrm{~nm}$ and $400 \mathrm{~nm}$ from the fundamental frequency and second harmonic of the Ti:sapphire laser.

Figure 2 (a) shows the experimental results for the efficiency in argon and a fitting $\lambda^{-5.9}$ line, consistent with the scaling relation for driver wavelengths in the near and mid-IR regime. Figure 2 (b) shows the experimental data for the cut-off energy in helium as black squares, while the red dashed line shows the linear fitting curve in logarithmic scale $\left(\lambda^{1.7 \pm 0.2}\right)$, which stays within the range of the phase-matched cut-off relation observed in the infrared region [4]. The absolute value of the cut-off energy is not optimized due to the low energy of the OPA driver pulses. To show the cut-off energy improvement with higher pulse energies, we numerically studied HHG by solving the 3-D propagation of the driver pulse with the three-step model HHG. Figure 2 (b) shows also a simulation of the cut-off energy in the experimental conditions (blue triangles and connecting line) and one with saturation of the cut-off energy, occurring with few $\mathrm{mJ}$ driver energies (green circles and connecting line, see also Fig. 2 (c)). The simulations show that with more pulse energy than what is possible with the current OPA, one could reach higher cut-off energies. To show the improvement in efficiency with higher pulse energies, we simulated the efficiency for three different He gas jet pressures, as shown in Figure 2 (c). As the pulse energy increases from $120 \mu \mathrm{J}$ to few mJs, the simulated efficiency improves by 2 or 3 orders of magnitude.
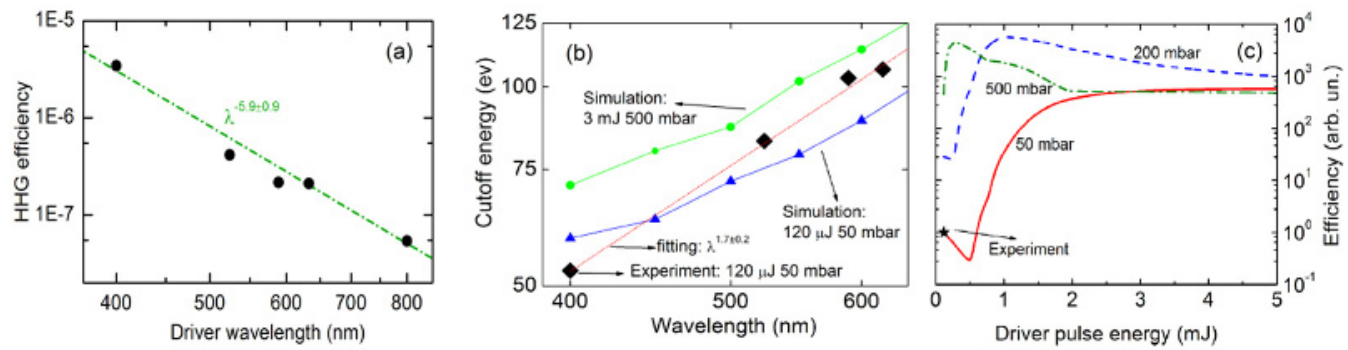

Fig. 2. (a) efficiency scaling in argon; (b) cut-off energy scaling in helium, experimental points and simulations; (c) calculated HHG efficiencies in helium at higher driving energies than the experiments.

1. G. Lambert et al., New. J. Phys. 11, 083033 (2009)

2. B. Shan and Z. Chang, Phys. Rev. A 65, 011804 (2001)

3. P. Tzankov, J. Zheng, M. Mero, D. Polli, C. Manzoni and G. Cerullo, Opt. Lett. 31, 3629 (2006)

4. M. C. Chen et al., Phys. Rev. Lett. 105, 173901 (2010) 